\title{
Future OFDM-based Communication Systems Towards 6G and Beyond: Machine Learning Approaches
}

\author{
Filbert H. Juwono ${ }^{1^{*}}$, Regina Reine ${ }^{2}$ \\ ${ }^{1}$ Department of Electrical and Computer Engineering, Curtin University, Malaysia \\ ${ }^{2}$ Twigx Research, 71 - 75 Shelton Street, London WC2H 9JQ, United Kingdom \\ *Correspondence: filbert@ieee.org
}

SUBMITTED: 14 October 2021; REVISED: 9 November 2021; ACCEPTED: 10 November 2021

\begin{abstract}
The vision towards 6G communication networks demands higher transmission rates, massive amounts of data processing, and low-latency communication. Orthogonal Frequency Division Modulation (OFDM) has been adopted in the current $5 \mathrm{G}$ networks and has become one of the potential candidates for the future $6 \mathrm{G}$ and beyond communication systems. Although OFDM offers many benefits including high spectrum efficiency and high robustness against the multipath fading channels, it has major challenges such as frequency offset and high Peak-to-Average Power Ratio (PAPR). In order to deal with the increasingly complex communication network, Machine Learning (ML) has been increasingly utilised to create intelligent and more efficient communication network. The role of ML in dealing with frequency offset and high PAPR is discussed in this paper. In addition, ML techniques may be utilized for channel estimation, M2M networks, and biomedical applications. Finally, this paper discusses the challenges and benefits of incorporating ML into OFDM-based communication systems.
\end{abstract}

KEYWORDS: OFDM; 6G; machine learning; cyber-physical social system

\section{Introduction}

The fifth generation $(5 \mathrm{G})$ of communication has revolutionized the relationship between technology, individuals, and society, which leads to the new philosophy of Society 5.0 [1]. At the same time, the cyber-physical social system (CPSS) concept emerges, where everything is connected and data-driven [2]. In CPSS, there are three types of communication: humans to humans, humans and machines, and machines to machines (M2M) [3]. In an M2M network, sensors are strategically positioned throughout the environment, resulting in enormous data communication where processing data at high data rates becomes a challenging task. Intelligent and efficient methods are required to satisfy the standards of the future communication network.

Existing 5G technologies are unable to handle the massive amounts of data that must be transmitted in the future. As a result, researchers have begun developing the sixth generation (6G) of communication technologies. The following characteristics are desired in the 6G and beyond networks [4]: intelligent, high energy efficiency, and always-on global coverage. The data rate and end-to-end latency can be up to $1 \mathrm{~Tb} / \mathrm{s}$ and $1 \mathrm{~ms}$, respectively. $6 \mathrm{G}$ and beyond 
communication systems are often considered green communication systems due to the utilization of low-power devices [5]. OFDM has been proposed as the modulation waveform for $6 \mathrm{G}$ and beyond networks due to its flexibility in merging with other technologies [6,7]. In OFDM-based communication systems, the use of artificial intelligence techniques such as machine learning (ML) and deep learning (DL) is unavoidable. It has been demonstrated in the literature that ML may increase system performance when compared to a conventional system. As a result, in this paper, we will examine the role of ML in OFDM-based communication systems.

The rest of this paper will be structured as follows. Section 2 will briefly discuss the OFDM-based communication systems and their challenges. Section 3 presents the impacts of utilizing ML in OFDM-based communications. Section 4 presents the challenges in implementing ML in OFDM-based communications. Finally, a conclusion is presented in Section 5.

\section{OFDM-based Communication Systems and Its Challenges}

The fundamental principle of OFDM is to divide a stream of high-rate data into many parallel low-rate data streams, which are mapped to a number of orthogonal subcarriers. The spectrums of the OFDM subcarriers overlap with each other without introducing interference.

Consider the transmitter part of an OFDM system as illustrated in Fig. 1, where the total bandwidth, $\mathrm{W}$, is divided into $\mathrm{N}$ subcarriers. The modulated symbol for the kth subcarrier is represented as bk, with the symbol interval Ts. The spectrums of the OFDM subcarriers overlap with each other without introducing interference. A Digital OFDM system can be implemented by employing inverse Discrete Fourier Transform (IDFT) at the transmitter and DFT at the receiver.

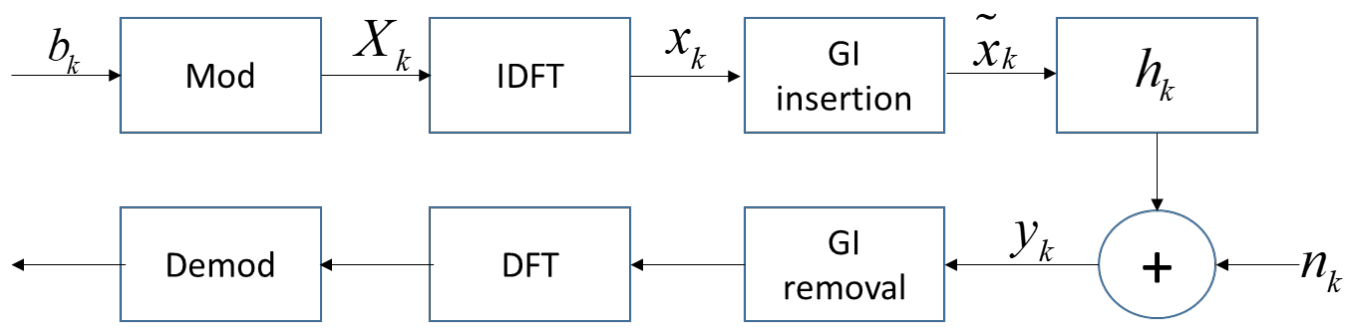

Figure 1. OFDM system.

The bit stream is passed to the modulation block ( $M$-QAM or $M$-PSK, where $M$ is the modulation order) and subsequently passed to the IDFT block, i.e., to form the OFDM signal. The analog baseband of the OFDM transmitted signal can be written as

$$
x_{k}=\sum_{k=0}^{N-1} b_{k} e^{j 2 \pi k t / T}, 0 \leq t \leq T .
$$

Guard interval (GI) may be appended to the OFDM signal to eliminate intersymbol interference (ISI), resulting in a longer sequence $\tilde{x}_{k}$. GI can be in the form of either a cyclic prefix or zero padding. The OFDM signal is then transmitted over the communication channel and received by the receiver. The received signal is expressed by 


$$
y_{k}=\tilde{x}_{k} * h_{k}+n_{k}
$$

where $h_{k}$ is the channel impulse response (CIR) coefficients, $n_{k}$ is the additive noise, and * denotes convolution. After the GI is removed, the received OFDM signal is passed to the DFT and demodulation blocks, respectively. In general, OFDM-based communication systems have two major challenges: frequency offset and a high peak-to-average power ratio in the transmitted OFDM signal.

\subsection{Frequency Offset}

Due to the orthogonality between the OFDM subcarriers, the receiver can demodulate the transmitted data without introducing inter-carrier interference (ICI). However, frequency mismatch (frequency offset) between the transmitter and receiver causes the carriers to become no longer orthogonal [8]. Frequency offset results in system performance degradation in terms of signal-to-noise ratio (SNR) and hence bit error rate (BER).

The strict orthogonality and synchronization in the OFDM system will restrict the capability of M2M communication where sporadic traffic is generated by the Internet of Things (IoT) or other devices in the M2M network. This type of device should not be forced to be integrated into the strict synchronization procedure. Ideally, these devices should wake occasionally, and then transmit their messages in an instance without strict synchronization. Future communications systems, such as $6 \mathrm{G}$ communication systems, are envisioned to connect devices over the air, underwater, and in space. Hence, more efficient techniques in channel estimation to minimize frequency offset are required.

\subsection{High Peak to Average Power Ratio}

Another major challenge in OFDM-based communication is the large PAPR in the transmitted signal. PAPR is an essential performance parameter of wireless communication systems as it reflects the cost and energy efficiency of the hardware requirements. Power efficiency is always an important factor in mobile communication networks to keep the total operational cost low. High PAPR of the transmitted OFDM signal has been one of the major drawbacks in OFDM system design and implementation. The large peaks resulting from high PAPR require additional back-off to prevent nonlinearity. Nonlinearity will create intermodulation between carriers and introduce signal distortion, thereby deteriorating the BER. The additional back-off means a larger dynamic range. This type of HPA is costly and consumes more power for portable systems, such as IoT devices. This is a major impediment to implementing OFDM for portable appliances. With the significant increase of utilised IoT devices in various systems, reducing the PAPR of the OFDM transmitted signal has been a popular topic in wireless communication.

Various conventional methods have been proposed to reduce the PAPR in OFDM communication systems, such as clipping [9], tone reservation [10,11], and pulse shaping [12]. However, these techniques have various limitations in minimizing the PAPR. 


\section{Machine Learning in OFDM based Communications}

Data-intensive applications over low-power devices will be the future of the fully connected world. In CPSS, data and humans become the focus of the technology where data will be transmitted and collected from machines and devices and humans. Technology will create a connected world where the collected data is used to solve society's problems. ML-OFDM has been proposed as an intelligent and efficient solution to various issues in communication systems.

\subsection{ML for Channel Estimation}

The use of deep learning (DL) has been proposed to be used at the OFDM receiver [13]. Training datasets are created offline under various environmental conditions where the received signals are recorded and used as training data for the system to learn. Furthermore, ML can be used to estimate channel impulse response (CIR) coefficients for equalization purposes [14]. In the future, communication using the OFDM signal will be transmitted over the air, space, or underwater channels to create a fully connected world. Underwater channel characteristics are different from radio frequency (RF) as underwater channels have different scenarios compared to air or space. Underwater acoustic (UWA) communications are used for surveillance systems, sensors, or unmanned vehicles underwater [1]. The underwater environment is complex, yet reliability and a high data rate are required for reliable communications. OFDM-ML can improve the BER performance in UWA compared to the existing optimization method [15].

\subsection{ML for Frequency Offset Estimation}

As discussed in [16], support vector machines (SVMs) have been employed to estimate the frequency offset in the demodulation process as well. It was shown that the system with SVM could achieve relatively the same BER as the maximum likelihood (ML) demodulator. Symbol timing offset, in addition to carrier offset, can produce phase distortion and intersymbol interference (ISI). SVM was proposed by the authors of [17] to tackle the symbol timing offset. The proposed method was demonstrated to be capable of estimating the timing offset, albeit the performance is dependent on the number of pilots.

\subsection{ML for PAPR Reduction}

An autoencoder of deep learning, called the PAPR reducing network (PRNet), was used as both a modulator and a demodulator to both reduce PAPR and achieve an undeteriotating BER in [18]. In particular, the loss function of PRNet was designed to meet the minimum PAPR as well as undeteriotating BER. Simulation results showed that PRNet outperforms traditional clipping and partial transmit scheme (PTS) methods. To efficiently reduce the PAPR, a lowcomplexity extreme machine learning (ELM)-based tone reservation was proposed in [19]. Compared to PRNet, the PAPR of ELM-based tone reservation is higher due to its low complexity. However, it can achieve a better symbol error rate (SER). [20] also proposed another DL-based tone reservation technique. Furthermore, ML techniques have also been used to lower out-of-band (OOB) emissions. A DL-based technique in terms of autoencoder has been employed for PAPR reduction [21]. A combination of extended SLM and autoencoder 
has also been proposed for reducing PAPR in DC-biased optical OFDM systems [22]. In [23], autoencoders together with orthogonal precoding were used to achieve both low PAPR and OOB emission. In [24], ML was used to improve the performance of clipping and filtering to reduce the PAPR of the OFDM signals.

\subsection{ML for M2M Networks}

Future communication systems should have flexibility, not strict orthogonality and synchronization, where different applications can be addressed by a single solution with different parameter settings instead of using multiple solutions, able to control the interference among multiple users and maintain the relationship with other technologies [25-27].

Implementing a large number of IoT devices in an M2M network requires an efficient and effective infrastructure to detect failure. Phenomena called "sleeping cells" can reduce the performance of the cell without triggering any warning and can be undetected by the system. In [28], the authors proposed using an ML framework to detect this type of failure efficiently.

\subsection{ML for Biomedical}

$6 \mathrm{G}$ technology has been envisioned to support the Internet of BioNano-Things (IoBNT) to provide reliable health monitoring and diagnostic systems with nano-bio sensors, machine learning, and molecular communications (MC) concepts. In [29], the authors discussed the visibility of diagnostic systems based on biomarker detection using molecular communication principles with an ML algorithm.

\section{Challenges in Machine Learning in OFDM based Communications}

ML in OFDM-based communication shows potential to improve various performances and is effective in reducing inefficiencies in order to satisfy the standards of future communication systems. However, implementing ML is complex, and many of the current approaches rely on dynamic training models. Producing high-quality training models requires high computational cost, and different parameters in the training models will produce different results. Training models require a large number of datasets, and sensitive data may be uploaded to the cloud server. Sensitive data may be stored in a central location and may be accessed inappropriately. This may lead to privacy and security issues. In time, the datasets may rapidly increase in size, and this will affect the speed of the processing, which will cause an issue for M2M networks where low latency is required. Most current ML algorithms and training models mainly use centralized servers, where super powerful servers are a necessity. Servers with super high computational speed can be costly, and it is one of the major restrictions for researchers and small-medium businesses.

\section{Conclusion}

For future $6 \mathrm{G}$ and beyond communication systems, embedding ML in OFDM-based communication will improve the performance and efficiency. ML has been used to solve some issues in OFDM-based communication systems, including frequency offset estimation, PAPR reduction, and channel estimation. It is also suitable for $\mathrm{M} 2 \mathrm{M}$ networks and biomedical engineering. However, there are challenges such as computational complexity, computational 
cost, distribution, security, privacy, and hardware cost in implementing ML in the communication system that need to be tackled.

\section{Competing Interest}

The authors declare no financial or non-financial competing interests.

\section{References}

[1] Deguchi, A.; Hirai, C.; Matsuoka, H.; Nakano, T.; Oshima, K.; Tai, M.; Tani, S. (2020). What is society 5.0?. In Society 5.0; Hitachi-U Tokyo Laboratory, Eds. Springer: Singapore, pp. 1-23. https://doi.org/10.1007/978-981-15-2989-4 1.

[2] Reine, R.; Juwono, F.H.; Sim, Z.A.; Wong, W.K. (2021). Cyber-physical-social systems: An overview. In Smart Connected World; Jain, S.; Murugesan, S., Eds; Cham: Springer, pp. 25-45. https://doi.org/10.1007/978-3-030-76387-9 2.

[3] Zhou, Y.; Liu, L.; Wang, L.; Hui, N.; Cui, X.; Wu, J.; Peng, Y.; Qi, Y.; Xing, C. (2020). Serviceaware 6G: An intelligent and open network based on the convergence of communication, computing, and caching. Digital Communications and Networks, 6, 253-260. https://doi.org/10.1016/j.dcan.2020.05.003.

[4] Letaief, K.B.; Chen, W.; Shi, Y.; Zhang, J.; Zhang, Y.A. (2019). The roadmap to 6G: AI empowered wireless networks. IEEE Communications Magazine, 57, 84-90. https://doi.org/10.1109/MCOM.2019.1900271.

[5] Verma, S.; Kaur, S.; Khan, M.A.; Sehdev, P.S. (2021). Toward green communication in 6Genabled massive internet of things. IEEE Internet Things Journal, 8, 5408-5415. https://doi.org/10.1109/JIOT.2020.3038804.

[6] Lee, Y.L.; Qin, D.; Wang, L.C.; Sim, G.H. (2021). 6G massive radio access networks: Key applications, requirements and challenges. IEEE Open Journal of Vehicular Technology, 2, 5466. https://doi.org/10.1109/OJVT.2020.3044569.

[7] Lu, W.; Si, P.; Liu, X.; Li, B.; Liu, Z.; Zhao, N.; Wu, Y. (2020). OFDM based bidirectional multirelay SWIPT strategy for 6G IoT networks. China Communications, 17, 80-91. https://doi.org/10.23919/JCC.2020.12.006.

[8] Lee, J.; Lou, H.L.; Toumpakaris, D.; Cioffi, J.M. (2004). Effect of carrier frequency offset on OFDM systems for multipath fading channels. IEEE Global Telecommunications Conference, 3721-3725. https://doi.org/10.1109/GLOCOM.2004.1379064.

[9] Tang, B.; Qin, K.; Mei H.; Chen, C. (2019). Iterative Clipping-Noise Compression Scheme for PAPR Reduction in OFDM Systems. IEEE Access, 7, 134348-134359. https://doi.org/10.1109/ACCESS.2019.2941389.

[10] Jiang, T.; Ni, C.; Ye, C.; Wu Y.; Luo, K. (2015). A Novel Multi-Block Tone Reservation Scheme for PAPR Reduction in OQAM-OFDM Systems. IEEE Transactions on Broadcasting, 61, 717 722. https://doi.org/10.1109/TBC.2015.2465146.

[11] Ni, C.; Ma, Y.; Jiang, T. (2016). A Novel Adaptive Tone Reservation Scheme for PAPR Reduction in Large-Scale Multi-User MIMO-OFDM Systems. IEEE Wireless Communications Letters, 5, 480-483. https://doi.org/10.1109/LWC.2016.2588489.

[12] Sim, Z.A.; Reine, R.; Zang, Z.; Juwono F. H.; Gopal, L. (2019). Reducing the PAPR of GFDM Systems with Quadratic Programming Filter Design. 2019 IEEE 89th Vehicular Technology Conference (VTC2019-Spring), 1-5. https://doi.org/10.1109/VTCSpring.2019.8746602.

[13] Ye, H.; Li, G.Y.; Juang, B.H. (2018). Power of deep learning for channel estimation and signal detection in OFDM systems. IEEE Wireless Communications Letters, 7, 114-117. https://doi.org/10.1109/LWC.2017.2757490.

[14] Jebur, B.A.: Alkassar, S.H.; Abdullah, M.A.M.; Tsimenidis, C.C. (2021). Efficient machine learning-enhanced channel estimation for OFDM systems. IEEE Access, 9, 100839-100850. https://doi.org/10.1109/ACCESS.2021.3097436.

[15] Kim, Y.; Lee, H.; Ahn, J.; Chung, J. (2019). Selection of CDMA and OFDM using machine learning in underwater wireless networks. ICT Express, 5, 215-218. https://doi.org/10.1016/j.icte.2019.09.002. 
[16] Siyari, P.; Rahbari, H.; Krunz, M. (2019). Lightweight machine learning for efficient frequencyoffset-aware demodulation. IEEE Journal on Selected Areas in Communications, 37, 2544-2558. https://doi.org/10.1109/JSAC.2019.2933956.

[17] Somarouthu, S.; Manam, S.; Thakre, A. (2020). Symbol detection in presence of symbol timing offset using machine learning technique. $20205^{\text {th }}$ IEEE International Conference on Recent Advances and Innovations in Engineering, 1-6. https://doi.org/10.1109/ICRAIE51050.2020.9358360.

[18] Kim, M.; Lee, W.; Cho, D.H. (2018). A novel PAPR reduction scheme for OFDM system based on deep learning. IEEE Communication Letters, 22, 510-513. https://doi.org/10.1109/LCOMM.2017.2787646.

[19] Li, Z.; Jin, N.; Wang, X.; Wei, J. (2021). Extreme learning machine-based tone reservation scheme for OFDM systems. IEEE Wireless Communication Letters, 10, 30-33, 2021. https://doi.org/10.1109/LWC.2020.3019792.

[20] Li, L.; Tellambura, C.; Tang, X. (2019). Improved tone reservation method based on deep learning for PAPR reduction in OFDM system. $201911^{\text {th }}$ International Conference on Wireless $\begin{array}{llll}\text { Communications and Signal Processing } & \text { (WCSP), }\end{array}$ https://doi.org/10.1109/WCSP.2019.8928103.

[21] Vahdat, M.; Roshandeh, K.P.; Ardakani, M.; Jiang, H. (2020). PAPR Reduction Scheme for Deep Learning-Based Communication Systems Using Autoencoders. 2020 IEEE $91^{\text {st }}$ Vehicular Technology Conference (VTC2020-Spring), 1-5. https://doi.org/10.1109/VTC2020Spring48590.2020.9128513.

[22] Hao, L.; Wang, D.; Tao, Y.; Cheng, W.; Li, J.; Liu, Z. (2019). The Extended SLM Combined Autoencoder of the PAPR Reduction Scheme in DCO-OFDM Systems. Applied Sciences, 9, 852. https://doi.org/10.3390/app9050852.

[23] Kuwahara R.; Ohta, M. (2020). PAPR and OOBE suppression of OFDM signal using deep learning. IEEE Global Conference on Consumer Electronics, 905-906. https://doi.org/10.1109/GCCE50665.2020.9291876.

[24] Sohn I.; Kim, S.C. (2015). Neural Network Based Simplified Clipping and Filtering Technique for PAPR Reduction of OFDM Signals. IEEE Communication Letters, 19, 1438-1441. https://doi.org/10.1109/LCOMM.2015.2441065.

[25] Lin, J.; Yu, W.; Zhang, N.; Yang, X.; Zhang, H.; Zhao, W. (2017). A survey on Internet of Things: Architecture enabling technologies security and privacy and applications. IEEE Internet Things Journal, 4, 1125-1142. https://doi.org/10.1109/JIOT.2017.2683200.

[26] Malandra, F.; Chiquette, L.O.; Lafontaine-Bédard, L.P.; Sansò, B. (2018). Traffic characterization and LTE performance analysis for M2M communications in smart cities. Pervasive and Mobile Computing, 48, 59-68. https://doi.org/10.1016/j.pmcj.2018.05.006.

[27] Shafiq, M.Z.; Ji, L.; Liu, A.X.; Pang, J.; Wang, J. (2012). A first look at cellular machine-tomachine traffic: Large scale measurement and characterization. ACM SIGMETRICS Performance Evaluation Review, 40, 65-76. https://doi.org/10.1145/2318857.2254767.

[28] Manzanilla-Salazar, O.G.; Malandra, F.; Mellah, H.; Wetté, C.; Sansò, B. (2020). A Machine Learning Framework for Sleeping Cell Detection in a Smart-City IoT Telecommunications Infrastructure. IEEE Access, 8, 61213-61225. https://doi.org/10.1109/ACCESS.2020.2983383.

[29] Juwono, F.H.; Reine, R.; Wong, W.K.; Sim, Z.A.; Gopal, L. (2021). Envisioning 6G Molecular Communication for IoBNT Diagnostic Systems. 2021 International Conference on Green Energy, Computing, and Sustainable Technology (GECOST), 1-5. https://doi.org/10.1109/GECOST52368.2021.9538653.

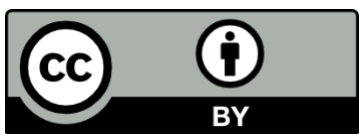

(C) 2021 by the authors. This article is an open access article distributed under the terms and conditions of the Creative Commons Attribution (CC BY) license (http://creativecommons.org/licenses/by/4.0/). 